\title{
Segmental resection of distal ureter with termino-terminal ureteric anastomosis vs bladder cuff removal and ureteric re-implantation for upper tract urothelial carcinoma: results of a multicentre study
}

\author{
Alberto Abrate* (D), Francesco Sessa", Arcangelo Sebastianelli", Mirko Preto* , \\ Alberto Olivero ${ }^{\S}$, Virginia Varca", Andrea Benelli", Riccardo Campi ${ }^{\dagger}$ (D), Maurizio Sessa**, ${ }^{*}$, \\ Carlo Pavone*, Vincenzo Serretta*, Marco Vella*, Eugenio Brunocilla $\$$, Sergio Serni ${ }^{\dagger}$, \\ Carlo Trombetta ${ }^{\S}$, Carlo Terrone ${ }^{\S}$, Andrea Gregori", Andrea Lissiani ${ }^{\S}$, Paolo Gontero , \\ Riccardo Schiavina ${ }^{+1}$, Mauro GaccitiD and Alchiede Simonato*(D)

\begin{abstract}
*Section of Urology, Department of Surgical, Oncological and Oral Sciences, University of Palermo, Palermo, 'Department of Minimally-Invasive and Robotic Urologic Surgery and Kidney Transplantation, Careggi Hospital, University of Florence, Florence, "Division of Urology, Department of Surgical Science, AOU Città della Salute e della Scienza di Torino - Presidio Molinette, University of Turin, Turin, ${ }^{\S}$ Department of Urology, IRCCS AOU San Martino, * *Department of Experimental Medicine, University of Campania 'L. Vanvitelli', Naples, Italy, "'Department of Drug Design and Pharmacology, University of Copenhagen, Copenhagen, Denmark, "tDepartment of Urology, St. OrsolaMalpighi Hospital, University of Bologna, Bologna, and ${ }^{\& s}$ Department of Urology, University of Trieste, Trieste, Italy
\end{abstract} \\ University of Genoa, Genoa, "Department of Urology, ASST Rhodense, G. Salvini Hospital, Garbagnate Milanese, Milan,
}

\section{Objectives}

To compare overall (OS), cancer-specific (CSS), recurrencefree survival (RFS) and postoperative renal function amongst patients with upper tract urothelial carcinoma (UTUC) of the distal (lower lumbar and pelvic) ureter, electively treated with segmental resection and termino-terminal anastomosis (TT) vs bladder cuff removal and ureteric re-implantation (RR).

\section{Patients and methods}

A multicentre retrospective study, including 84 patients diagnosed with UTUC of the distal ureter and treated with TT or RR, is presented. The primary endpoint was to compare TT and RR in terms of OS, CSS and RFS. As a secondary outcome, we compared the postoperative creatinine values as an index of renal function in the two groups.

\section{Results}

Of 521 patients with UTUC, 65 (77.4\%) and 19 (22.6\%) patients underwent RR and TT, respectively. Pre- and postoperative characteristics were not statistically different between the two groups. The median follow-up period was 22.7 months. Patients treated with TT and those treated with RR did not have significantly different 5-year OS, CSS or RFS (73.7\% vs $92.3 \%, P=0.052 ; 94.7 \%$ vs $95.4 \%, P=0.970$ : and $63.2 \%$ vs $53.9 \%, P=0.489$, respectively). No difference in postoperative creatinine variation emerged in association with the surgical technique $(P=0.411)$.

\section{Conclusion}

Patients treated with TT or RR for UTUC showed comparable OS, CSS, RFS and postoperative renal function. Our data suggest that bladder cuff removal is not imperative in the treatment of distal ureteric UTUC, and TT can be a safe solution in selected cases.

\section{Keywords}

renal function, segmental ureterectomy, survival, terminoterminal ureteroureterostomy, upper tract urothelial carcinoma, ureteric re-implantation, \#utuc

\section{Introduction}

Upper tract urothelial carcinoma (UTUC) is a rare malignancy $[1,2]$ that can be located in the pyelocalyceal cavities and, although less commonly, in the ureter [3]. Despite the fact that radical nephroureterectomy (RNU) with bladder cuff removal is currently considered as the standard treatment [4], recently kidney-sparing surgery (KSS), and in 
particular open or laparoscopic segmental ureterectomy (SU), has been proposed in selected cases such as solitary kidney, chronic kidney disease and synchronous bilateral disease, together with endoscopic and percutaneous procedures [5].

It is commonly accepted in reconstructive surgery that short and uncomplicated proximal ureteric defects can be treated with direct ureteroureterostomy, whilst ureteroneocystostomy or ureteroureterostomy can be performed for distal ones, guarantying a tension-free repair [6].

Short-term overall (OS), cancer-specific (CSS) and recurrence-free survival (RFS) in a recent meta-analysis in patients treated for UTUC did not significantly differ between SU and RNU [7]. Moreover, RNU can be associated with a significant decrease in renal function in comparison with SU. Data for survival and functional outcomes are missing for long-term follow-up and even less is known about functional outcomes and survival amongst patients undergoing different surgical techniques of ureteric anastomosis after SU for UTUC.

Considering this gap in the literature, the aim of our present study was to compare the OS, CSS and RFS amongst patients undergoing surgery for UTUC, electively treated with segmental resection of the distal ureter and termino-terminal anastomosis (TT) vs bladder cuff removal and ureteric reimplantation (RR). As a secondary endpoint, the impact of the surgical technique on postoperative renal function was evaluated.

\section{Patients and methods}

\section{Study design}

Data from patients treated for UTUC in six Italian tertiary referral centres (Bologna, Genoa, Milan, Palermo, Trieste, Turin), from January 2003 to December 2013, were collected. All consecutive patients who underwent TT or RR for UTUC of the distal (lower lumbar and pelvic) ureter, were included in the analysis. All patients were diagnosed with UTUC at CT or MRI. Preoperative ureteroscopy with biopsy was performed when indicated by inconclusive imaging. Other preoperative data included: age, gender, smoking status, creatinine levels $(\mathrm{mg} / \mathrm{dL})$, symptoms, urine cytology, tumour site, hydronephrosis, stage and grade of previous bladder tumour. Patients with a history of previous UTUC, metastatic disease, radical cystectomy or other malignancies were excluded. Patients were treated with SU and TT or RR according to tumour location. Pathological data included: $\mathrm{T}$ and $\mathrm{N}$ category, carcinoma in situ (CIS) detection, grade, number of lymph nodes dissected, lymph vascular invasion, tumour necrosis, surgical margin status, and the presence of concomitant bladder cancer. Patients underwent follow-up every 3 months in the first year after surgery, every 4 months in the second year, every 6 months from the third to the fifth year, and then annually, according to the most recent international guidelines [8]. Serum creatinine levels, recurrence, and the necessity of chemotherapy were recorded.

\section{Statistical analysis}

Statistical analysis was performed by one of the authors (M.S.). Patient characteristics were compared at baseline and at the end of the follow-up period, using the Student's $t$-test or Mann-Whitney $U$-test for continuous variables and chisquared for categorical variables. The primary outcome of the present study was to compare TT and RR in terms of OS, CSS and RFS. Thus, unadjusted 5-year OS curves of the two groups were compared using the Kaplan-Meier method, whilst 5-year CSS and RFS were compared using the cumulative incidence method [9]. A multivariate Cox regression adjusted for technique, age, gender, chemotherapy and recurrence was used to estimate hazard ratios (HRs). As a secondary outcome, we investigated the $\Delta$ creatinine (postoperative minus preoperative creatinine) in the two groups. In particular, a multivariate Cox regression adjusted for technique, age, gender, tumour side and localisation, hydronephrosis, tumour pathological stage, chemotherapy and preoperative creatinine was used to calculate the HR. Post hoc power analyses for unadjusted analyses were performed by using power calculation methods described by Freedman [10], Schoenfeld [11] and Latouche et al. [12]. In particular, we computed if with our study sample we were able to observe an HR of 8.7, 0.01, and 0.05 for 5-year OS, CSS, and RFS between TT and RR, respectively. Statistical analyses were performed with $\mathrm{R}$ version 3.3.3. A $P<0.05$ was considered as statistically significant.

\section{Results}

We retrospectively collected the data of 521 patients treated for UTUC, from 2003 to 2013, in six selected Italian centres. Overall, 84 patients underwent segmental resection of the ureter for a distal ureteric UTUC; 65 (77.4\%) and 19 (22.6\%) patients underwent RR and TT, respectively. For preoperative characteristics, only the localisation of the tumour and the performance of an endoscopic biopsy prior to surgery showed statistically different distributions amongst patients treated with RR and TT $(P<0.001$ and $P=0.046$, respectively; Table 1). Overall only four cases of perioperative complications were reported: three Clavien-Dindo Grade II (fever requiring antibiotics) and one Grade III (intravesical bleeding requiring endoscopic treatment). Four (three after $\mathrm{RR}$ and one after TT) ascertained anastomotic strictures were reported, but endoscopic correction was required in only two of them.

Postoperative pathological characteristics including: stage, grade, number of lymph nodes dissected, lymphovascular invasion, necrosis, positive surgical margins, and presence of 
Table 1 Preoperative descriptive characteristics of the study population.

\begin{tabular}{|c|c|c|c|c|}
\hline Variable & Total & $\mathbf{R R}$ & $\pi$ & $\boldsymbol{P}$ \\
\hline Patients, $n(\%)$ & $84(100)$ & $65(77.4)$ & $19(22.6)$ & \\
\hline Age, years, mean (sD) & $69.5(9.1)$ & $69.8(9.7)$ & $68.7(6.8)$ & 0.651 \\
\hline \multicolumn{5}{|l|}{ Gender, $n(\%)$} \\
\hline Male & $65(77.4)$ & $52(80.0)$ & $13(68.4)$ & \multirow[t]{2}{*}{0.454} \\
\hline Female & $19(22.6)$ & $13(20.0)$ & $6(31.6)$ & \\
\hline \multicolumn{5}{|l|}{ Smoking status (30 patients), $n$ (\%) } \\
\hline No & $12(40.0)$ & $11(45.8)$ & $1(16.7)$ & \multirow[t]{2}{*}{0.402} \\
\hline Yes & $18(60.0)$ & $13(54.2)$ & $5(83.3)$ & \\
\hline Preoperative serum creatinine, $\mathrm{mg} / \mathrm{dL}$, mean (SD) & $1.04(0.37)$ & $1.04(0.39)$ & $1.05(0.34)$ & 0.964 \\
\hline \multicolumn{5}{|l|}{ Symptoms (43 patients), $n(\%)$} \\
\hline No & $15(34.9)$ & $11(32.4)$ & $4(44.4)$ & \multirow[t]{2}{*}{0.777} \\
\hline Yes & $28(65.1)$ & $23(67.6)$ & $5(55.6)$ & \\
\hline \multicolumn{5}{|l|}{ Prior endoscopic biopsy, $n$ (\%) } \\
\hline No & $65(77.4)$ & $54(83.1)$ & $11(57.9)$ & \multirow[t]{2}{*}{0.046} \\
\hline Yes & $19(22.6)$ & $11(16.9)$ & $8(42.1)$ & \\
\hline Negative & $4(21.1)$ & $3(27.3)$ & $1(12.5)$ & \multirow[t]{2}{*}{0.834} \\
\hline Positive & $15(78.9)$ & $8(72.7)$ & $7(87.5)$ & \\
\hline \multicolumn{5}{|l|}{ Prior urine cytology, $n(\%)$} \\
\hline No & $64(76.2)$ & $50(76.9)$ & $14(73.7)$ & \multirow[t]{2}{*}{0.988} \\
\hline Yes & $20(23.8)$ & $15(23.1)$ & $5(26.3)$ & \\
\hline Negative & $12(60.0)$ & $11(73.3)$ & $1(20.0)$ & \multirow[t]{2}{*}{0.114} \\
\hline Positive & $8(40.0)$ & $4(26.7)$ & $4(80.0)$ & \\
\hline \multicolumn{5}{|l|}{ Tumour localisation, $n$ (\%) } \\
\hline Intramural & $27(32.1)$ & $27(41.5)$ & $0(0.0)$ & \multirow[t]{3}{*}{$<0.001$} \\
\hline Pelvic & $46(54.8)$ & $33(50.8)$ & $13(68.4)$ & \\
\hline Lower lumbar & $11(13.1)$ & $5(7.7)$ & $6(31.6)$ & \\
\hline \multicolumn{5}{|l|}{ Tumour side, $n(\%)$} \\
\hline Right & $31(36.9)$ & $26(40.0)$ & $5(16.3)$ & \multirow[t]{2}{*}{0.414} \\
\hline Left & $53(63.1)$ & $39(60.0)$ & $14(73.7)$ & \\
\hline \multicolumn{5}{|l|}{ Hydronephrosis (44 patients), $n$ (\%) } \\
\hline No & $11(25.0)$ & $11(32.4)$ & $0(0.0)$ & \multirow[t]{2}{*}{0.097} \\
\hline Yes & $33(75.0)$ & $23(67.6)$ & $10(100.0)$ & \\
\hline \multicolumn{5}{|l|}{ History of bladder tumour, $n(\%)$} \\
\hline No & $54(64.3)$ & $40(61.5)$ & $14(73.7)$ & \multirow[t]{2}{*}{0.484} \\
\hline Yes & $30(35.7)$ & $25(38.5)$ & $5(26.3)$ & \\
\hline \multicolumn{5}{|l|}{ Prior bladder tumour $\mathrm{pT}$ stage (29 patients), $n$ (\%) } \\
\hline $\mathrm{pTa}$ & $11(36.6)$ & $9(36.0)$ & $2(50.0)$ & \multirow[t]{3}{*}{0.663} \\
\hline pT1 & $14(46.6)$ & $12(48.0)$ & $2(50.0)$ & \\
\hline CIS & $4(13.8)$ & $4(16.0)$ & $0(0.0)$ & \\
\hline \multicolumn{5}{|l|}{ Prior bladder tumour grade (28 patients), $n$ (\%) } \\
\hline Grade 1 & $7(25.0)$ & $5(20.8)$ & $2(50.0)$ & \multirow[t]{3}{*}{0.285} \\
\hline Grade 2 & $8(28.6)$ & $8(33.3)$ & $0(0.0)$ & \\
\hline Grade 3 & $13(46.4)$ & $11(45.8)$ & $2(50.0)$ & \\
\hline
\end{tabular}

concomitant bladder cancer, were not statistically different between the groups (Table 2).

The median (range) follow-up of the study population was 22.7 (0.5-120) months. Overall, nine (10.7\%) and 24 $(28.6 \%)$ patients had local ureteric and bladder recurrence, respectively; whilst four $(4.8 \%)$ patients developed metastatic disease, showing a comparable distribution between the groups $(P=0.061$, Table 3$)$. There were no statistically significant differences in terms of 5-year OS, CSS and RFS amongst patients treated with TT and RR (73.7\% vs $92.3 \%, P=0.052 ; 94.7 \%$ vs $95.4 \%, P=0.970$; and $63.2 \%$ vs $53.9 \%, P=0.489$; respectively) (Fig. 1). Post hoc power analyses revealed that for expected HRs we had a statistical power of 0.80 . At the multivariable adjusted analysis, the type of reconstructive technique, gender and age were not significantly associated with OS, CSS and RFS. Postoperative chemotherapy was the only significant determinant for CSS with a HR of 9.86 (95\% CI 1.3174.42, $P=0.03$; Fig. S1).

There was no statistically significant difference in postoperative creatinine variation amongst patients treated with TT and RR [mean (SD) $\Delta$ creatinine $0.0(0.3)$ and 0.1 (0.2) $\mathrm{mg} / \mathrm{dL}$, respectively; $P=0.383$ ]. In the adjusted analysis, statistically significant determinants of postoperative creatinine variation were preoperative creatinine level (HR $0.35,95 \%$ CI $0.10-0.60 ; P=0.010$ ), male gender (HR 0.31, 95\% CI 0.10-0.52; $P=0.006$ ), left-side location (HR 0.37, 95\% CI $0.09-0.64 ; P=0.012)$, and pathological T3 stage (pT3) disease (HR 0.50, 95\% CI 0.09-0.91; $P=0.020)$, irrespective of the surgical technique ( $P=0.411$; Fig. 2 ). 
Table 2 Pathological characteristics.

\begin{tabular}{|c|c|c|c|c|}
\hline Variable & Total & $\mathbf{R R}$ & $\pi$ & $\boldsymbol{P}$ \\
\hline Patients, $n$ (\%) & $84(100)$ & $65(77.4)$ & $19(22.6)$ & \\
\hline \multicolumn{5}{|l|}{ Pathological T-stage, $n(\%)$} \\
\hline pT0 & $2(2.4)$ & $1(1.5)$ & $1(5.3)$ & \multirow[t]{6}{*}{0.721} \\
\hline pTa & $30(35.7)$ & $21(32.3)$ & $9(47.4)$ & \\
\hline pT1 & $21(25.0)$ & $17(26.2)$ & $4(21.0)$ & \\
\hline pT2 & $19(22.6)$ & $16(24.6)$ & $3(15.8)$ & \\
\hline pT3 & $11(13.1)$ & $9(13.8)$ & $2(10.5)$ & \\
\hline CIS & $1(1.2)$ & $1(1.5)$ & $0(0.0)$ & \\
\hline \multicolumn{5}{|l|}{ CIS associated, $n(\%)$} \\
\hline No & $81(96.4)$ & $62(95.4)$ & $19(100.0)$ & \multirow[t]{2}{*}{0.802} \\
\hline Yes & $3(3.6)$ & $3(4.6)$ & $0(0.0)$ & \\
\hline \multicolumn{5}{|l|}{ Lymph node dissection, $n$ (\%) } \\
\hline No & $65(77.4)$ & $48(73.8)$ & $17(89.5)$ & \multirow[t]{2}{*}{0.262} \\
\hline Yes & $19(22.6)$ & $17(26.2)$ & $2(10.5)$ & \\
\hline No. lymph nodes dissected, mean (SD) & $1.1(2.9)$ & $1.4(3.2)$ & $0.1(0.2)$ & 0.089 \\
\hline \multicolumn{5}{|l|}{ Pathological N-stage, $n(\%)$} \\
\hline $\mathrm{pN} 0$ & $17(20.2)$ & $15(23.1)$ & $2(10.5)$ & \multirow[t]{3}{*}{0.334} \\
\hline $\mathrm{pN} 1$ & $2(2.4)$ & $2(3.1)$ & $0(0.0)$ & \\
\hline $\mathrm{pNx}$ & $65(77.4)$ & $48(73.8)$ & $17(89.5)$ & \\
\hline \multicolumn{5}{|l|}{ Grade (81 patients), $n$ (\%) } \\
\hline Grade 1 & $12(14.8)$ & $7(11.1)$ & $5(27.8)$ & \multirow[t]{3}{*}{0.173} \\
\hline Grade 2 & $21(25.9)$ & $16(25.4)$ & $5(27.8)$ & \\
\hline Grade 3 & $48(59.3)$ & $40(63.5)$ & $8(44.4)$ & \\
\hline \multicolumn{5}{|c|}{ Lymphovascular invasion (46 patients), $n$ (\%) } \\
\hline No & $42(91.3)$ & $32(91.4)$ & $10(90.9)$ & \multirow[t]{2}{*}{0.576} \\
\hline Yes & $4(8.7)$ & $3(8.6)$ & $1(9.1)$ & \\
\hline \multicolumn{5}{|l|}{ Necrosis (83 patients), $n$ (\%) } \\
\hline No & $80(96.4)$ & $62(96.9)$ & $18(94.7)$ & \multirow[t]{2}{*}{0.794} \\
\hline Yes & $3(3.6)$ & $2(3.1)$ & $1(5.3)$ & \\
\hline \multicolumn{5}{|l|}{ Surgical margins, $n(\%)$} \\
\hline Negative & $69(82.1)$ & $55(84.6)$ & $14(73.7)$ & \multirow[t]{2}{*}{0.451} \\
\hline Positive & $15(17.9)$ & $10(15.4)$ & $5(26.3)$ & \\
\hline \multicolumn{5}{|l|}{ Concomitant bladder cancer, $n$ (\%) } \\
\hline No & $67(79.8)$ & $49(75.4)$ & $18(94.7)$ & \multirow[t]{2}{*}{0.128} \\
\hline Yes & $17(20.2)$ & $16(24.6)$ & $1(5.3)$ & \\
\hline
\end{tabular}

Table 3 Postoperative follow-up data.

\begin{tabular}{|c|c|c|c|c|}
\hline Variable & Total & RR & $\pi$ & $\boldsymbol{P}$ \\
\hline Patients, $n(\%)$ & $84(100)$ & $65(77.4)$ & $19(22.6)$ & \\
\hline $\begin{array}{l}\text { Follow-up period, months, median } \\
(95 \% \text { confidence interval }[95 \% \mathrm{CI}])\end{array}$ & $22.7(14.0-29.0)$ & $21.5(12.2-33.3)$ & $25.2(12.4-39.9)$ & 0.769 \\
\hline Postoperative creatinine, $\mathrm{mg} / \mathrm{dL}$, mean $(\mathrm{SD})$ & $1.1(0.3)$ & $1.1(0.3)$ & $1.0(0.3)$ & 0.580 \\
\hline$\Delta$ creatinine, $\mathrm{mg} / \mathrm{dL}$, mean $(\mathrm{sD})$ & $0.1(0.3)$ & $0.1(0.2)$ & $0.0(0.3)$ & 0.383 \\
\hline \multicolumn{5}{|l|}{ Recurrence, $n$ (\%) } \\
\hline No & $47(56.0)$ & $35(53.8)$ & $12(63.2)$ & 0.648 \\
\hline Yes & $37(44.0)$ & $30(46.2)$ & $7(36.8)$ & \\
\hline Ipsilateral & $7(18.9)$ & $3(10.0)$ & $4(57.1)$ & 0.061 \\
\hline Contralateral & $1(2.7)$ & $1(3.3)$ & $0(0.0)$ & \\
\hline Bilateral & $1(2.7)$ & $1(3.3)$ & $0(0.0)$ & \\
\hline Vesical & $24(64.9)$ & $22(73.3)$ & $2(28.6)$ & \\
\hline Metastasis & $4(10.8)$ & $3(10.0)$ & $1(14.3)$ & \\
\hline \multicolumn{5}{|l|}{ Systemic chemotherapy, $n$ (\%) } \\
\hline No & $76(90.5)$ & $59(90.8)$ & $17(89.5)$ & 0.783 \\
\hline Yes & $8(9.5)$ & $6(9.2)$ & $2(10.5)$ & \\
\hline
\end{tabular}

Abbreviations: CIS, carcinoma in situ; CSS, cancer-specific survival; HR, hazard ratio; KSS, kidney-sparing surgery; OS, overall survival; pT, pathological T stage; recurrence-free survival; $R R$, bladder cuff removal and ureteric re-implantation; $R N U$, radical nephroureterectomy; TT, segmental resection of the distal ureter and termino-terminal anastomosis; SU, segmental ureterectomy; UTUC, upper tract urothelial carcinoma.

\section{Discussion}

In this multicentre retrospective study, we compared the OS, CSS and RFS of two different ureteric reconstruction techniques after SU for distal ureteric UTUC. Interestingly, patients treated with TT and RR showed comparable 5-year OS, CSS and RFS.

Although it is known that up to $70 \%$ of ureteric tumours occur in the distal ureter, $25 \%$ in the mid-ureter, and $5 \%$ in 
Fig. 1 Comparison between RR and TT techniques in terms of 5-year OS (A), CSS (B) and RFS (C).

A

RR vs TT

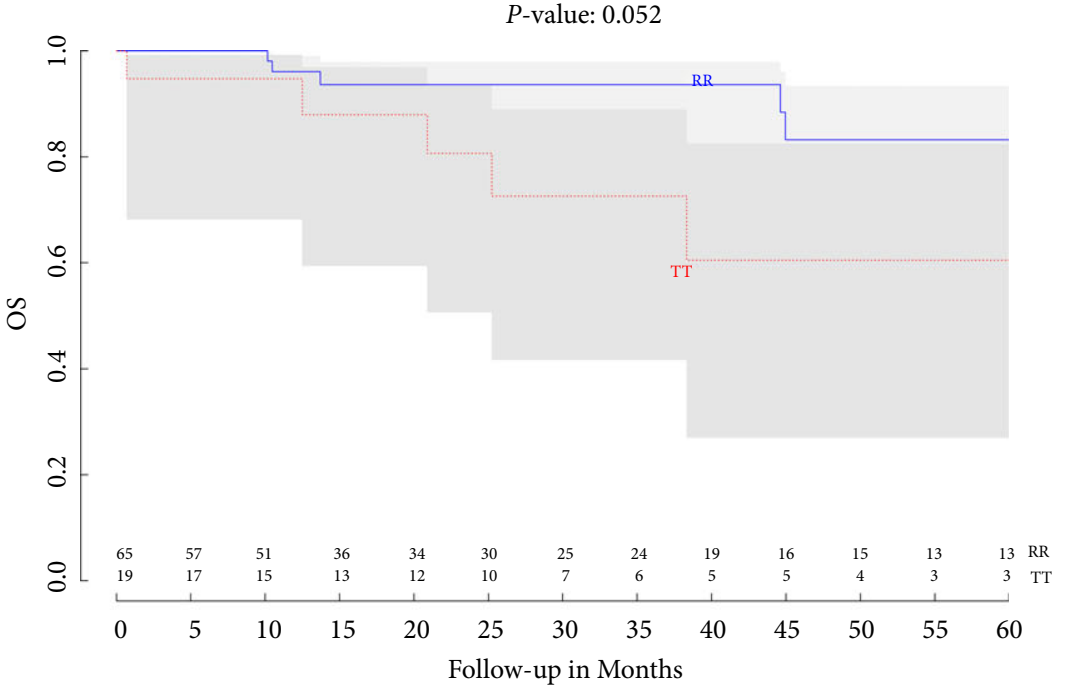

B

RR vs TT

$P$-value: 0.970

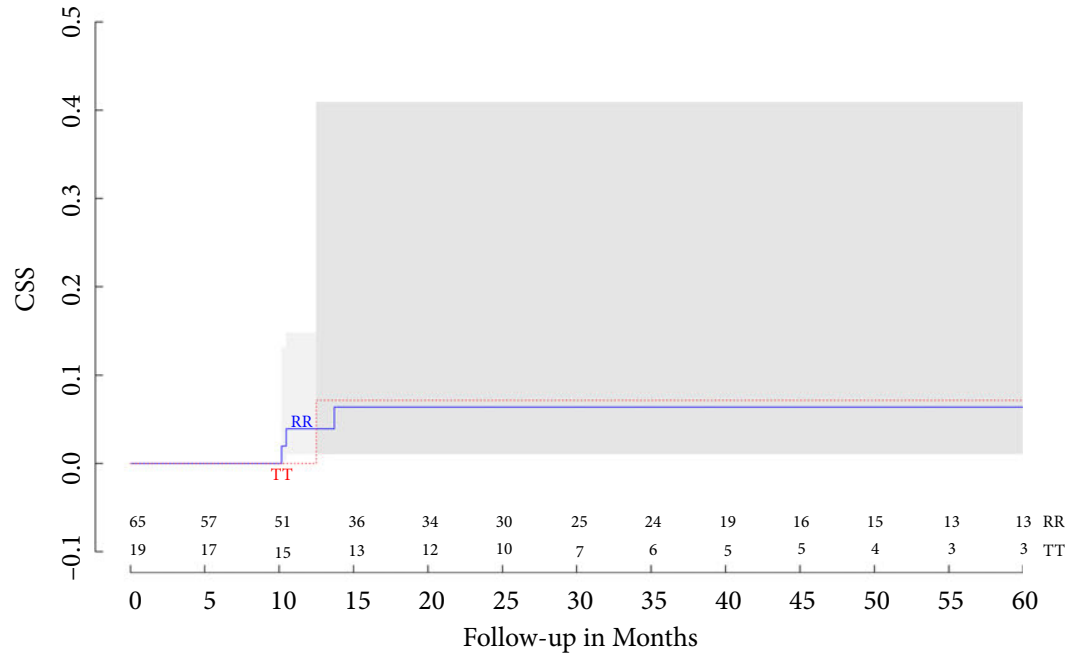

C

RR vs TT

$P$-value: 0.489

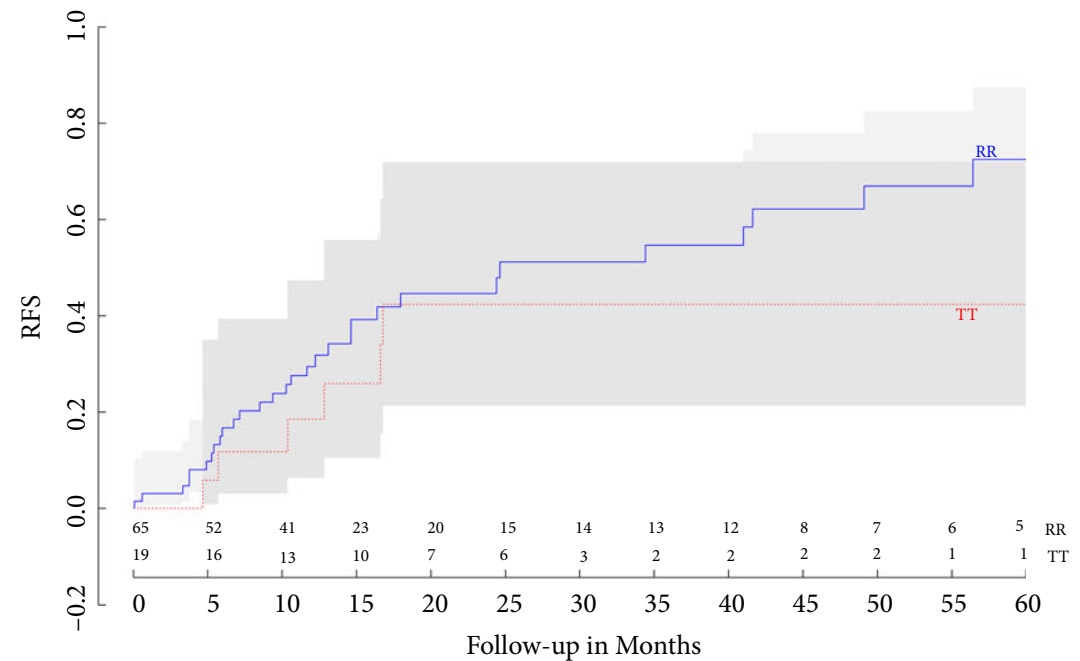


Fig. 2 Multivariate Cox regression adjusted analysis of postoperative creatinine variation.

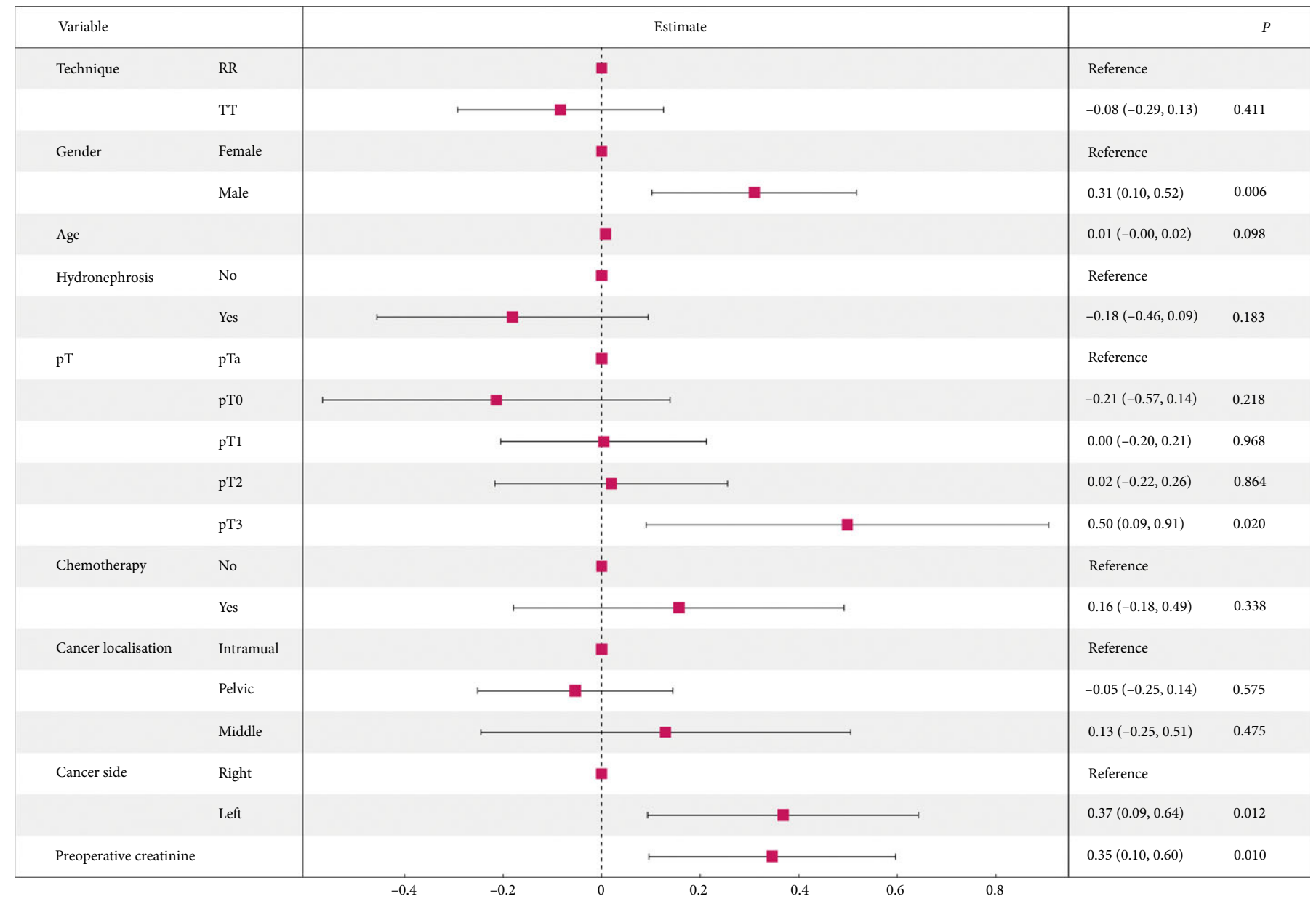

the proximal ureter [13], RNU is widely indicated as the standard treatment for UTUC, regardless of tumour localisation [4], and the removal of the entire ureter seems to be mandatory. In fact, it has been discussed that total excision of the distal ureter with its intramural portion, including the ipsilateral ureteric orifice and bladder cuff, is essential for optimal treatment of UTUC [14]. Clearly the rationale for the indication of excising all the affected upper urinary tract derives from the observation of a high disease recurrence rate in the remaining ureteric stump after a simple nephrectomy [15].

However, chronic kidney disease has been reported in $52 \%$ of patients with UTUC at diagnosis, with this rate significantly increasing to $78 \%$ after RNU, with a median relative reduction in renal function of $21 \%$ [16] and an estimated GFR decrease of $9.32 \mathrm{~mL} / \mathrm{min} / 1.73 \mathrm{~m}^{2}(P=0.007)$ after RNU in comparison with SU [7]. Today, KSS is acceptable in selected cases of anatomical or functional solitary kidney, bilateral disease or severe renal insufficiency to avoid the morbidities frequently occurring following RNU [17]. KSS and RNU have been compared on heterogeneous patients' populations, and the available data are therefore highly variable and difficult to adapt to common clinical practice [18].

Recently, a meta-analysis published by Fang et al. [7] in 2016 found no significant differences between SU and RNU in terms of CSS (unadjusted HR 0.90, 95\% CI 0.73-1.11; $P=0.33$ ). Although some concerns have been raised about the dissimilar distribution of $\mathrm{T}$ stage and grade between the two treatment groups, no significant differences were detected in terms of CSS in patients with non-muscleinvasive UTUC [19] and high-grade disease [20]. Also OS (HR 0.98, 95\% CI 0.63-1.53; $P=0.93$ ) and RFS (HR 1.06, 95\% CI $0.76-1.48 ; P=0.72$ ) appear to be comparable between SU and RNU [7].

Translating the experience from RNU to KSS, it is common practice to also excise the bladder cuff for distal ureteric UTUC with consequent ureteric re-implantation. However, it has not yet clearly been demonstrated if any differences exist 
for functional and oncological outcomes amongst patients treated with RR and TT for UTUC.

Both ureteroneocystostomy and ureteroureterostomy have been suggested with good outcomes for benign and malignant ureteric diseases. Wenske et al. [21] reported excellent functional outcomes without significant morbidity in a population of 100 patients undergoing ureteric reimplantation by psoas hitch, Boari flap, or ureteroneocystostomy for benign ureteric obstruction or UTUC, with no statistically significant difference between the three different surgical techniques. On the other hand, open end-to-end ureteroureterostomy was effective and safe in treating iatrogenic lower ureteric injury at a median follow-up of 33.7 months [22]. A ureteric patency rate of $96 \%$ has also been reported in patients treated with open ureteroneocystostomy or ureteroureterostomy with no significant difference adopting a laparoscopic approach $(P=0.544)$ at a mean follow-up of 43 months [23]. Moreover, ureteroureterostomy showed significantly less estimated blood loss $(P<0.001)$ and a lower incidence of VUR (Grade I) on cystography $(P=0.031)$ in comparison with ureteroneocystostomy at a follow-up of 36.5 months [24].

In the case of UTUC, different reconstruction techniques are often analysed all together and, to our knowledge, there are no comparative studies between RR and TT in terms of oncological outcomes. Interestingly, in our present experience TT and RR showed similar 5-year OS, CSS and RFS rates (Fig. 1), better than those after RNU (data not shown), with a low rate of ureteric (10.7\%) and bladder (28.6\%) recurrence (Table 3). Accordingly, a 5-year OS of 40-72\%, 5-year CSS of $54-90 \%$, 5-year RFS of 28-84\%, and an intravesical RFS of $54-69 \%$ have been reported in patients treated with SU for UTUC [7].

There is no consensus on the role of tumour location in terms of oncological outcomes for ureteric cancer: in our experience the choice of the reconstructive technique primarily depends on tumour location and this is reflected in the different distribution in the two groups (RR vs TT). This could also explain the different (although not significant) distribution of recurrence reported in the two groups.

We also detected a comparable distribution of postoperative pT stage and grade, reducing the concern about selection bias. Noteworthy, the rate of positive surgical margin was similar between the groups, with a slightly higher rate in the RR group, contrary to popular belief.

Considering the postoperative variation of renal function in terms of serum creatinine levels, we found that preoperative creatinine, male gender, pT3 stage and left-side were associated with a significant HR for postoperative creatinine variation. This observation could be partially explained considering that in our present population male patients and those with left-sided tumours also had higher preoperative creatinine levels (although not statistically significantly). Conversely, pT stage had already been associated to renal function [25].

Finally, the main limitation of our present study is its retrospective design and the small sample size, partially due to the rarity of the disease. Consequently, some probably interesting data, such as the dimensions of the disease at CT or previous intravesical chemotherapy, were not available. No pathological data on concomitant or recurrent bladder cancer and chemotherapy protocol were available. Another limitation is the lack of data on administration of postoperative instillation after surgery at the Centres included in our present study. On the contrary the main strength of our present study is its multicentre approach with a validated and shared follow-up scheme. An exhaustive comparison between RNU and SU, in terms of oncological and functional outcomes, was beyond the intents of the present study.

In conclusion, we report that patients treated with TT or RR for UTUC showed comparable 5-year OS, CSS and RFS. Moreover, no significant differences were found in terms of postoperative creatinine variation between the two surgical techniques. Our present results suggest that bladder cuff removal is not imperative in the treatment of distal ureteric UTUC, and TT can be a safe solution when feasible in selected cases.

\section{Acknowledgement}

Special thanks to Lisa Marie Terzariol for the linguistic revision.

\section{Funding}

Dr Maurizio Sessa postdoc is supported by a grant from the Novo Nordisk Foundation to the University of Copenhagen (NNF15SA0018404).

\section{Conflicts of Interest}

None declared.

\section{References}

1 Munoz JJ, Ellison LM. Upper tract urothelial neoplasms: incidence and survival during the last 2 decades. J Urol 2000; 164: 1523-5

2 Siegel RL, Miller KD, Jemal A. Cancer statistics, 2017. CA Cancer J Clin 2017; 67: 7-30

3 Cosentino M, Palou J, Gaya JM, Breda A, Rodriguez-Faba O, Villavicencio-Mavrich $\mathrm{H}$. Upper urinary tract urothelial cell carcinoma: location as a predictive factor for concomitant bladder carcinoma. World J Urol 2013; 31: 141-5

4 Margulis V, Shariat SF, Matin SF et al. Outcomes of radical nephroureterectomy: a series from the Upper Tract Urothelial Carcinoma Collaboration. Cancer 2009; 115: 1224-33 
5 Seisen T, Peyronnet B, Dominguez-Escrig JL et al. Oncologic outcomes of kidney-sparing surgery versus radical nephroureterectomy for upper tract urothelial carcinoma: a systematic review by the EAU Non-muscle Invasive Bladder Cancer Guidelines Panel. Eur Urol 2016; 70: 1052-68

6 Png JC, Chapple CR. Principles of ureteric reconstruction. Curr Opin Urol 2000; 10: 207-12

7 Fang D, Seisen T, Yang K et al. A systematic review and meta-analysis of oncological and renal function outcomes obtained after segmental ureterectomy versus radical nephroureterectomy for upper tract urothelial carcinoma. Eur J Surg Oncol 2016; 42: 1625-35

8 Roupret M, Babjuk M, Comperat E et al. European Association of Urology Guidelines on Upper Urinary Tract Urothelial Carcinoma: 2017 update. Eur Urol 2018; 73: 111-22

9 Zhang Z. Survival analysis in the presence of competing risks. Ann Transl Med 2017; 5: 47

10 Freedman LS. Tables of the number of patients required in clinical trials using the logrank test. Stat Med 1982; 1: 121-9

11 Schoenfeld DA. Sample-size formula for the proportional-hazards regression model. Biometrics 1983; 39: 499-503

12 Latouche A, Porcher R, Chevret S. Sample size formula for proportional hazards modelling of competing risks. Stat Med 2004; 23: 3263-74

13 Smith AK, Matin SF, Jarrett TW. Urothelial tumors of the upper urinary tract and ureter. In Wein AJ, Kavoussi LR, Partin AW, Peters CA eds, Campbell-Walsh Urology, 11th edn, Chpt 58. Philadelphia, PA: Elsevier, 2016: 1365-1402

14 Lucca I, Leow JJ, Shariat SF, Chang SL. Diagnosis and management of upper tract urothelial carcinoma. Hematol Oncol Clin North Am 2015; 29 : 271-88, ix

15 Hall MC, Womack S, Sagalowsky AI, Carmody T, Erickstad MD, Roehrborn CG. Prognostic factors, recurrence, and survival in transitional cell carcinoma of the upper urinary tract: a 30-year experience in 252 patients. Urology 1998; 52: 594-601

16 Lane BR, Smith AK, Larson BT et al. Chronic kidney disease after nephroureterectomy for upper tract urothelial carcinoma and implications for the administration of perioperative chemotherapy. Cancer 2010; 116: 2967-73

17 Gakis G, Schubert T, Alemozaffar M et al. Update of the ICUD-SIU consultation on upper tract urothelial carcinoma 2016: treatment of localized high-risk disease. World J Urol 2017; 35: 327-35

18 Seisen T, Colin P, Roupret M. Risk-adapted strategy for the kidney-sparing management of upper tract tumours. Nat Rev Urol 2015; 12: 155-66

19 Colin P, Ouzzane A, Pignot G et al. Comparison of oncological outcomes after segmental ureterectomy or radical nephroureterectomy in urothelial carcinomas of the upper urinary tract: results from a large French multicentre study. BJU Int 2012; 110: 1134-41

20 Bagrodia A, Kuehhas FE, Gayed BA et al. Comparative analysis of oncologic outcomes of partial ureterectomy vs radical nephroureterectomy in upper tract urothelial carcinoma. Urology 2013; 81: 972-7

21 Wenske S, Olsson CA, Benson MC. Outcomes of distal ureteral reconstruction through reimplantation with psoas hitch, Boari flap, or ureteroneocystostomy for benign or malignant ureteral obstruction or injury. Urology 2013; 82: 231-6

22 Paick JS, Hong SK, Park MS, Kim SW. Management of postoperatively detected iatrogenic lower ureteral injury: should ureteroureterostomy really be abandoned? Urology 2006; 67: 237-41

23 Simmons MN, Gill IS, Fergany AF, Kaouk JH, Desai MM. Laparoscopic ureteral reconstruction for benign stricture disease. Urology 2007; 69: 280-4

24 Wang Z, Chen Z, He Y, Li B, Wen Z, Chen X. Laparoscopic ureteroureterostomy with an intraoperative retrograde ureteroscopyassisted technique for distal ureteral injury secondary to gynecological surgery: a retrospective comparison with laparoscopic ureteroneocystostomy. Scand J Urol 2017; 51: 329-34

25 Fang D, Zhang Q, Li X et al. Nomogram predicting renal insufficiency after nephroureterectomy for upper tract urothelial carcinoma in the Chinese population: exclusion of ineligible candidates for adjuvant chemotherapy. Biomed Res Int 2014; 2014: 529186 https://doi.org/10.1155/ 2014/529186

Correspondence: Alchiede Simonato, Section of Urology, Department of Surgical, Oncological and Oral Sciences, University of Palermo, via del Vespro 129, 90127 Palermo, Italy.

e-mail: alchiede@gmail.com

\section{Supporting Information}

Additional Supporting Information may be found in the online version of this article:

Figure S1. Multivariate Cox regression adjusted analysis for OS (A), CSS (B) and RFS (C). 